OPEN ACCESS

Edited by:

Laurence Morel,

University of Florida, United States

Reviewed by:

Weici Zhang,

University of California, Davis,

United States

Pietro Invernizzi

University of Milano-Bicocca, Italy

*Correspondence:

Gary L. Norman

glnorman@inovadx.com

Specialty section:

This article was submitted to Autoimmune and Autoinflammatory

Disorders,

a section of the journal

Frontiers in Immunology

Received: 17 December 2018

Accepted: 11 March 2019

Published: 03 April 2019

Citation:

Norman GL, Reig A, Viñas O, Mahler M, Wunsch E, Milkiewicz P.

Swain MG, Mason A, Stinton LM,

Aparicio MB, Aldegunde MJ,

Fritzler MJ and Parés A (2019) The

Prevalence of Anti-Hexokinase-1 and Anti-Kelch-Like 12 Peptide Antibodies

in Patients With Primary Biliary

Cholangitis is Similar in Europe and North America: A Large International,

Multi-Center Study.

Front. Immunol. 10:662.

doi: 10.3389/fimmu.2019.00662

\section{The Prevalence of Anti-Hexokinase-1 and Anti-Kelch-Like 12 Peptide Antibodies in Patients With Primary Biliary Cholangitis Is Similar in Europe and North America: A Large International, Multi-Center Study}

\author{
Gary L. Norman ${ }^{1 *}$, Anna Reig ${ }^{2}$, Odette Viñas ${ }^{3}$, Michael Mahler ${ }^{1}$, Ewa Wunsch ${ }^{4}$, \\ Piotr Milkiewicz ${ }^{4,5}$, Mark G. Swain ${ }^{6}$, Andrew Mason ${ }^{7}$, Laura M. Stinton ${ }^{6}$, \\ Maria Belen Aparicio ${ }^{8}$, Maria Jose Aldegunde ${ }^{8}$, Marvin J. Fritzler ${ }^{6}$ and Albert Parés ${ }^{2}$ \\ ${ }^{1}$ Department of Research and Development, Inova Diagnostics, San Diego, CA, United States, ${ }^{2}$ Liver Unit, Hospital Clínic, \\ Institut D'Investigacions Biomèdiques August Pi i Sunyer, CIBERehd, University of Barcelona, Barcelona, Spain, ${ }^{3}$ Immunology \\ Department, Hospital Clínic, Centre Diagnòstic Biomèdic, Institut D'Investigacions Biomèdiques August Pi i Sunyer, \\ Barcelona, Spain, ${ }^{4}$ Translational Medicine Group, Pomeranian Medicine University, Szczecin, Poland, ${ }^{5}$ Liver and Internal \\ Medicine Unit, Department General, Transplant and Liver Surgery, Medical University of Warsaw, Warsaw, Poland, ${ }^{6}$ Cumming \\ School of Medicine, University of Calgary, Calgary, AB, Canada, ${ }^{7}$ Division of Gastroenterology (Liver Unit), University of \\ Alberta, Edmonton, $A B$, Canada, ${ }^{8}$ Laboratorio Autoimmunidad, Hospital Universitario de Salamanca, Salamanca, Spain
}

Primary biliary cholangitis (PBC), formerly known as primary biliary cirrhosis, is present worldwide. Autoantibodies, in particular anti-mitochondrial antibodies (AMA) detected by indirect immunofluorescence assays or newer solid phase immunoassays can detect most, but not all individuals with PBC. Detection of antibodies to the anti-nuclear antigens sp100 and gp210 can identify additional PBC patients, but some seronegative patients remain, often resulting in delayed diagnosis and treatment. Antibodies to kelch-like 12 (KLHL12) and hexokinase 1 (HK-1) were recently identified as new biomarkers for PBC and notably identify patients who are negative for conventional autoantibodies. To become globally adopted, it is important to validate these new biomarkers in different geographic areas. In the present study we evaluated the prevalence of anti-KLHL12 (measured by a KLHL12-derived peptide referred to as KL-p) and anti-HK-1 antibodies by ELISA at five sites within Europe and North America and demonstrated the presence of these antibodies in patients with PBC in all geographies.

Keywords: primary biliary cholangitis, autoantibodies, anti-mitochondrial antibodies, AMA-negative, hexokinase1, kelch-like 12

\section{INTRODUCTION}

For over 50 years, the detection of anti-mitochondrial antibodies (AMA) on rodent tissue sections has remained the primary diagnostic biomarker for primary biliary cholangitis (PBC) in many laboratories, and when performed and interpreted at expert centers, the assay is highly sensitive and specific for PBC (1-3). It is clear however, that up to $15 \%$ of patients with clinically-proven 
PBC are AMA-negative by indirect immunofluorescence (IIF) (4-7). Using recombinant proteins such as M2 (MIT3), which incorporates the three immunodominant epitopes recognized by AMA, namely the E2 subunits of pyruvate dehydrogenase complex (PDC-E2), the branched chain 2oxo-acid dehydrogenase complex (BCOADC-E2), and the 2-oxo-glutareate dehydrogenase complex (OGDC-E2), can result in increased sensitivity and the ability to detect additional, but still not all, AMA-negative PBC patients $(4,8,9)$. While additional AMA-negative $\mathrm{PBC}$ patients can be identified by the presence of anti-sp100 corresponding to the multiple nuclear dot pattern by IIF on HEp-2 cells (AC-6 according to International Consensus of ANA patterns, ICAP), anti-gp210 punctate nuclear envelope by IIF on HEp-2 cells (AC-12 according to ICAP), and anti-p62 antibodies, some $\mathrm{PBC}$ patients remain seronegative, potentially resulting in delayed diagnosis and treatment (10-14).

Autoantibodies to kelch-like 12 protein (KLHL12) and to hexokinase $1(\mathrm{HK}-1)$ were recently identified as novel biomarkers in patients with $\mathrm{PBC}(15,16)$. In a cohort of 366 patients with PBC, $\sim 40$ and $45 \%$ of 277 AMA-positive patients were positive for anti-KLHL12 or anti-HK-1 antibodies respectively, while in 89 AMA-negative patients 53 and $42 \%$ were positive for antiKLHL12 or anti-HK-1 antibodies, respectively. The specificities of both antibodies was 96-97\% (15).

KLHL12 is part of a large, evolutionarily conserved superfamily consisting of $66 \mathrm{KLHL}$ genes (17). The various Kelch proteins appear to be involved in multiple cellular functions including cell structure, cellular communication, transcriptional regulation, collagen export, and ubiquitination of proteins through interaction with the E3-ligase cullin $(17,18)$. HK-1 is an enzyme which localizes to the outer membrane of mitochondria and phosphorylates glucose to yield glucose6-phosphate, as well as modulating cellular susceptibility to apoptosis (19).

Although difficult to clearly establish, it appears that the prevalence of PBC is increasing worldwide (20-22). While AMA has a similar prevalence in PBC patients from different geographies, the prevalence of anti-KLHL12 and anti-HK-1 antibodies in different geographic areas has not been reported. To address this issue, we examined PBC sera collected from patients in Eastern Europe, Western Europe, and Canada for the presence of anti-KLHL12 and anti-HK-1 antibodies.

\section{MATERIALS AND METHODS}

Sera from a total of 487 patients with clinically documented $\mathrm{PBC}$ or $\mathrm{PBC} / \mathrm{AIH}$ overlap diagnosed according to European Association for the Study of the Liver (EASL) guidelines were collected at five expert clinical sites (Barcelona, Spain; Salamanca, Spain; Calgary, Canada; Edmonton, Canada; Warsaw, Poland) and tested for the presence of autoantibodies to HK-1 and a KLHL12-derived immunodominant peptide, referred to as "KLp," using research use only ELISA kits (QUANTA Lite ${ }^{\circledR}$, Inova Diagnostics, San Diego, CA). All sera were also tested for antiM2 (MIT3) antibodies (subsequently referred to as anti-MIT3 for simplicity) by ELISA (QUANTA Lite ${ }^{\circledR}$ ELISA, Inova Diagnostics, San Diego, CA). Specificity was assessed by testing 127 sera from patients without $\mathrm{PBC}$, including patients with primary sclerosis cholangitis (PSC, $n=41$ ), autoimmune hepatitis (AIH, $n=20$ ), AIH/PSC overlap $(n=12)$, various infectious diseases $(n=20)$, colorectal cancer $(n=14)$, and healthy controls $(n=20)$.

Differences between the five cohorts was assessed by oneway analysis of variance (ANOVA) using Krustal-Wallis test and between specific geographic chorts using Mann-Whitney nonparametric two-tailed $t$-test (Graphpad ver 5.03, San Diego, CA). $P$-value $<0.05$ was considered significant.

\section{RESULTS}

Testing for anti-MIT3 antibodies showed that each cohort had different frequencies of anti-MIT3 negative individuals (Calgary, 7.2\%; Barcelona, 12.9\%, Edmonton, 16.3\%; Salamanca, 23.8\%, and Warsaw, 36.6\%) $(p=0.0002)$. Overall anti-HK-1 antibodies were found in $36.6-52.4 \%$ and anti-KL-p antibodies in from $22.0-33.3 \%$ of the cohorts. The specific prevalence of anti-HK-1 and KL-p antibodies at each site is summarized in Table $\mathbf{1 .}$

As a result of the apparent heterogeneity of the cohorts, we proceeded to examine the performance of the two biomarkers after specimens were stratified by anti-MIT3 positivity. Anti-HK1 antibody positivity in anti-MIT3-positive individuals ranged from $38.5-56.3 \%$ as detailed in Table 1. While the groups were statistically different overall (ANOVA $p=0.0006$ ), there was no difference between some of the sites, for example Barcelona and Edmonton $(p=0.8169)$. When all specimens from the five cohorts were combined, anti-HK-1 antibody was positive

TABLE 1 | Frequency of anti-HK-1, anti-KL-p, and anti-HK-1 and/or anti-KL-p in each geographic cohort and in a combined cohort including all patients.

Anti-HK-1+

Anti-KL-p+

Anti-HK-1+ and/or KL-p+

Total Cohort $N=$ No. (\%) MIT3+ No. (\%) MIT3- $P$ - value No. (\%) MIT3+ No. (\%) MIT3- $P$-value No. (\%) MIT3+ No. (\%) MIT3- $P$-value

\begin{tabular}{|c|c|c|c|c|c|c|c|c|c|c|}
\hline Barcelona & 224 & 75/195 (38.5) & 10/29 (34.5) & 0.8379 & 49/195 (25.1) & 3/29 (10.3) & 0.0991 & 103/195 (52.8) & 13/29 (44.8) & 0.4341 \\
\hline Calgary & 97 & 48/90 (53.3) & $1 / 7(14.8)$ & 0.0592 & 23/90 (25.6) & 2/7 (28.6) & 1.000 & $59 / 90(65.6)$ & $3 / 7$ (42.9) & 0.2486 \\
\hline Edmonton & 104 & $45 / 87(51.7)$ & $2 / 17$ (11.8) & 0.0028 & 22/87 (25.2) & 2/17 (11.8) & 0.3476 & $54 / 87(62.1)$ & 3/17 (17.6) & 0.0014 \\
\hline Warsaw & 41 & $12 / 26(46.2)$ & $3 / 15$ (20.0) & 0.1772 & $6 / 26(23.1)$ & 3/15 (20.0) & 1.000 & $15 / 26(57.7)$ & 5/15 (33.3) & 0.1971 \\
\hline Salamanca & 21 & 9/16 (56.3) & $2 / 5$ (40.0) & 0.6351 & 3/16 (18.8) & 4/5 (80.0) & 0.0251 & $9 / 16(56.2)$ & 4/5 (80.0) & 0.6065 \\
\hline COMBINED & 487 & $189 / 414(45.7)$ & 18/73 (24.7) & 0.0008 & $103 / 414$ (24.9) & 14/73 (19.2) & 0.3725 & $240 / 414$ (58.0) & 28/73 (38.4) & 0.0022 \\
\hline
\end{tabular}

P-values $<0.5$ are considered significant and indicated by bold type. 
in $45.7 \%(189 / 414)$ of the anti-MIT3-positive specimens, with a specificity of $94.5 \%$ (120/127). Of the seven non-PBC specimens that tested positive, four had a diagnosis of AIH, two colorectal cancer, and one was an apparently healthy control. The likelihood ratio (LR)+, LR-, positive predictive value (PPV), negative predictive value (NPV), and Odds Ratio (OR) of anti-HK-1 antibodies for the combined cohort of all anti-MIT3-positive specimens was $8.28,0.58,0.96,0.35$, and 14.40 .

Anti-KL-p antibody positivity in the anti-MIT3-positive specimens ranged from $18.8-25.6 \%$ (Table 1). Overall, when all specimens were combined, anti-KL-p antibody was positive in
$24.9 \%(103 / 414)$ of the anti-MIT3-positive specimens with a specificity of $95.3 \%(121 / 127)$. As with anti-HK-1 antibodies, while the groups were statistically different overall (ANOVA $p$ $<0.0001$ ), several showed no statistical difference, again for example Barcelona-Edmonton, Barcelona-Warsaw, EdmontonWarsaw ( $p=0.9973,0.4333$, and 0.0868 respectively). The LR+, LR-, PPV, NPV, and OR for anti-KL-p antibodies for the combined cohort of anti-MIT3-positive specimens was 4.60, 0.79, $0.96,0.18$, and 5.80 .

Examination of anti-MIT3-negative PBC patients in the five cohorts showed a prevalence of anti-HK-1 antibodies ranging

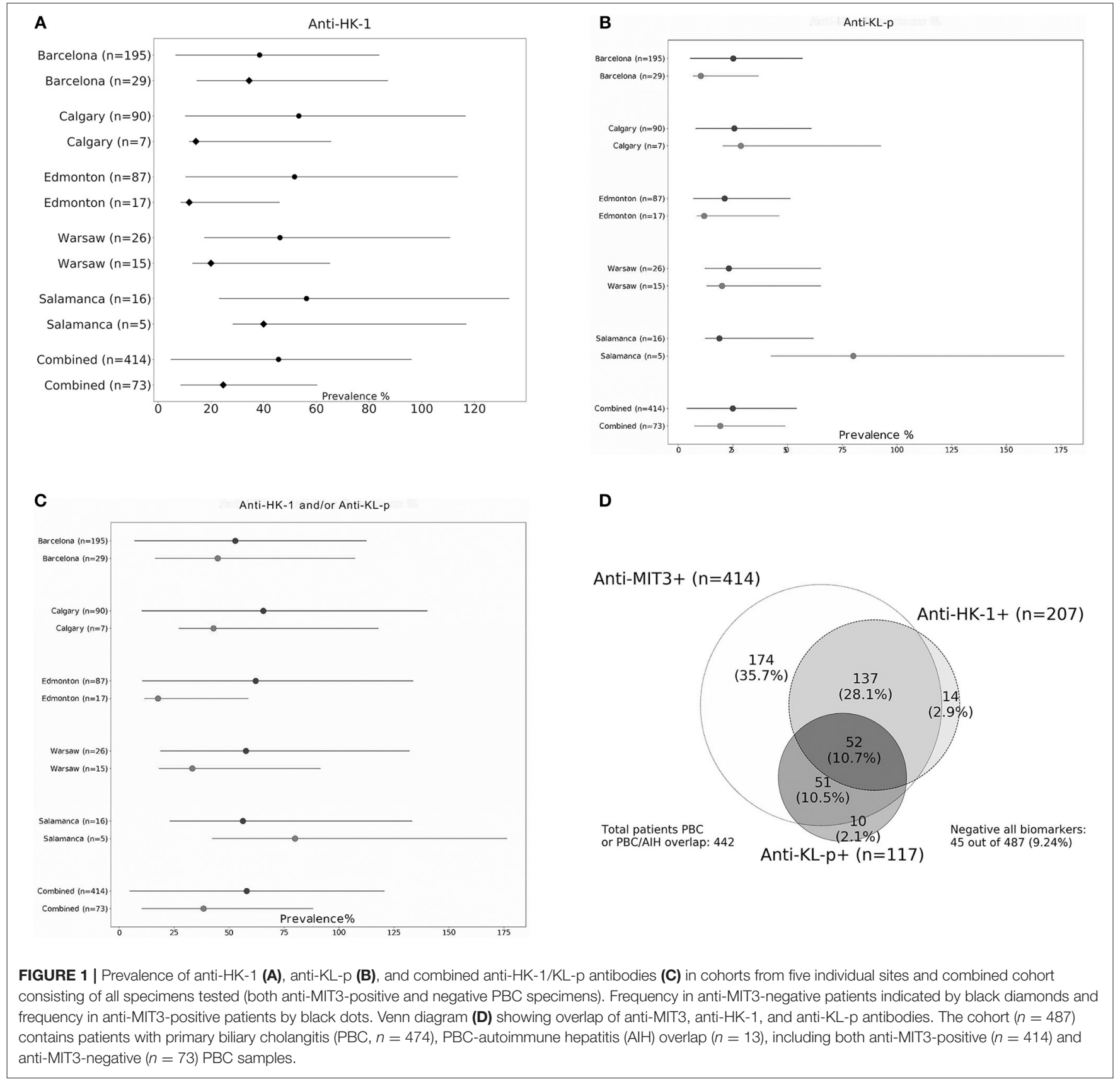


from $11.8-40.0 \%$ and anti-KL-p antibodies from 10.3 to $80.0 \%$ (Table 1). Among all anti-MIT3-negative specimens from the 5 sites, anti-HK-1 antibodies were found in 18/73 (24.7\%) and anti-KL-p in $14 / 73(19.2 \%)$ of the patients. Employing both markers to examine the anti-MIT3-negative patients increased the sensitivity to $38.4 \%(28 / 73)$ with a small decrease in specificity to $91.3 \%$.

The overlap of anti-MIT3, anti-HK-1, and anti-KL-p antibodies is illustrated using a Venn diagram in Figure 1. The addition of anti-HK-1 and anti-KL-p antibody testing to anti-MIT3 antibody results increased the sensitivity from $85.0 \%$ for anti-MIT3 alone to $90.8 \%$ for the combination of anti-MIT3, anti-HK-1, and anti-KL-p for the total study cohort.

\section{DISCUSSION}

The incidence of PBC, like many other autoimmune diseases, appears to be increasing worldwide (20-22). Environmental factors have been suggested as one potential contributing cause of the increasing incidence of PBC (3). In addition, better diagnostics and better recognition of the disease, especially during the often long asymptomatic phase, are also likely involved in the apparent increased prevalence $(23,24)$. The availability of new therapeutics such as obeticholic acid provides an additional rationale to identify patients with PBC early in the disease course, so that treatment and monitoring that might slow, or ideally prevent progression can be instituted (22). New biomarkers to help identify AMA or anti-MIT3-negative $\mathrm{PBC}$ patients who may otherwise remain unrecognized, are particularly important to help achieve this goal.

Despite the regular description of new biomarkers in the literature, only a few contribute additional actionable clinical value and are incorporated into routine clinical practice. We have previously shown that anti-HK-1 and anti-KL-p antibodies are present in both AMA-positive and, importantly, AMAnegative patients (15). Our current results demonstrate that the general prevalence of these autoantibodies is similar in 5 different sites examined from North America and Europe. The pooled prevalence of anti-HK-1 antibodies was $45.7 \%$ in anti-MIT3positive patients and $24.7 \%$ in anti-MIT3-negative patients. AntiKL-p antibodies were detected in $24.9 \%$ of the anti-MIT3positive patients and $19.2 \%$ of the anti-MIT3-negative patients. Combined testing for both antibodies found $58.0 \%$ of the antiMIT3-positive and $38.4 \%$ of the anti-MIT3-negative patients seropositive for one or both of the new antibodies. The new biomarkers therefore may help to close the serological gap in $\mathrm{PBC}$ patients. The prevalence of the markers appeared more

\section{REFERENCES}

1. Walker JG, Doniach D, Roitt IM, Sherlock S. Serological tests in diagnosis of primary biliary cirrhosis. Lancet. (1965) 1:827-31. doi: 10.1016/S0140-6736(65)91372-3

2. Beuers U, Gershwin ME, Gish RG, Invernizzi P, Jones DE, Lindor K, et al. Changing nomenclature for PBC: from 'cirrhosis' to 'cholangitis'. Hepatology. (2015) 62:1620-2. doi: 10.1002/hep.28140 similar in the anti-MIT3-positive patients from the different sites compared to the anti-MIT3-negative patients. This is reasonable since the anti-MIT3-positive patients are likely a more clinically homogeneous group compared to the anti-MIT3-negative group, which may have a more heterogeneous phenotype. The small sample size of several of the cohorts is also a clear limitation of the study and likely contributes to some of the perceived differences between cohorts. The higher prevalence of anti-HK-1 antibodies compared to anti-KL-p antibodies could be related to the presence of $\mathrm{HK}-1$ on the mitochondrial membrane, similar to other PBC-related antigens. In contrast, explanation why antibodies to KLHL12 are increased in patients with $\mathrm{PBC}$ remains intriguing, but still unclear at present.

Early treatment of patients with PBC appears beneficial and, therefore, addition of anti-KL-p and anti-HK-1 autoantibody testing has the potential to decrease delays in diagnosis and treatment (22). Although we did not examine the prevalence of the antibodies in Asian and South American populations, these studies are currently being organized. A key question remaining is whether, in addition to their value in diagnosis, these antibodies can be shown to be associated with particular clinical phenotypes or outcomes. Preliminary results, so far only presented in abstract form, suggest this may be the case for anti-HK-1 antibodies, where the presence of these antibodies at diagnosis was associated with significantly increased levels of alkaline phosphatase, alanine aminotransaminase, and gamma-glutamyl transpeptidase compared to anti-HK-1 negative patients, as well as with shorter transplant-free survival time (25). These results are potentially of important clinical interest, however additional validation and studies are now needed to confirm their significance.

\section{AUTHOR CONTRIBUTIONS}

GN, MM, AP, MS, AM, PM, MF, and AR contributed to the design of the study. OV, EW, MBA, MJA, and LS contributed to the acquisition and analysis of data. GN wrote the manuscript. All authors reviewed and approved the publication of the content.

\section{ACKNOWLEDGMENTS}

Portions of this study were presented September 2017 at the 13th Dresden Symposium on Autoantibodies, Dresden, Germany (26). We thank Zakera Shums, Jay Milo, Susan Encabo, Chelsea Bentow, Silvia Ruiz-Gaspà, Maria Antonia Romera, Mila Garcia, and Natalia Baeza for technical assistance with specimen handling and antibody measurements. We thank Carlos Melus for his assistance with preparation of figures. of primary biliary cirrhosis. Annu Rev Pathol. (2013) 8:303-30. doi: 10.1146/annurev-pathol-020712-164014

4. Miyakawa H, Tanaka A, Kikuchi K, Matsushita M, Kitazawa E, Kawaguchi N, et al. Detection of antimitochondrial autoantibodies in immunofluorescent AMA-negative patients with primary biliary cirrhosis using recombinant autoantigens. Hepatology. (2001) 34:243-8. doi: 10.1053/jhep.2001. 26514 
5. Muratori P, Muratori L. Autoantibodies in antimitochondrial antibody negative primary biliary cholangitis. Clin Res Hepatol Gastroenterol. (2017) 41:e47. doi: 10.1016/j.clinre.2017.04.016

6. Muratori P, Muratori L, Gershwin ME, Czaja AJ, Pappas G, MacCariello S, et al. 'True' antimitochondrial antibody-negative primary biliary cirrhosis, low sensitivity of the routine assays, or both? Clin Exp Immunol. (2004) 135:154-8. doi: 10.1111/j.1365-2249.2004.02332.x

7. Invernizzi P, Selmi C, Ranftler C, Podda M, Wesierska-Gadek J. Antinuclear antibodies in primary biliary cirrhosis. Semin Liver Dis. (2005) 25:298-310. doi: 10.1055/s-2005-916321

8. Moteki S, Leung PS, Coppel RL, Dickson ER, Kaplan MM, Munoz S, et al. Use of a designer triple expression hybrid clone for three different lipoyl domain for the detection of antimitochondrial autoantibodies. Hepatology. (1996) 24:97-103. doi: 10.1002/hep.510240117

9. Milkiewicz P, Buwaneswaran H, Coltescu C, Shums Z, Norman GL, Heathcote EJ. Value of autoantibody analysis in the differential diagnosis of chronic cholestatic liver disease. Clin Gastroenterol Hepatol. (2009) 7:1355-60. doi: 10.1016/j.cgh.2009.07.012

10. Chan EKL, Damoiseaux J, Carballo OG, Conrad K, de Melo Cruvinel W, Francescantonio PLC, et al. Report of the first international consensus on standardized nomenclature of antinuclear antibody HEp-2 cell patterns 2014-2015. Front. Immunol. (2015) 6:412. doi: 10.3389/fimmu.2015. 00412

11. Sebode M, Weiler-Normann C, Liwinski T, Schramm C. Autoantibodies in autoimmune liver disease-clinical and diagnostic relevance. Front Immunol. (2018) 9:609. doi: 10.3389/fimmu.2018.00609

12. Tartakovsky F, Worman HJ. Detection of gp210 autoantibodies in primary biliary cirrhosis using a recombinant protein containing the predominant autoepitope. Hepatology. (1995) 21:495-500.

13. Wesierska-Gadek J, Penner E, Battezzati PM, Selmi C, Zuin M, Hitchman E, et al. Correlation of initial autoantibody profile and clinical outcome in primary biliary cirrhosis. Hepatology. (2006) 43:1135-44. doi: 10.1002/hep.21172

14. Liu H, Norman GL, Shums Z, Worman HJ, Krawitt EL, Bizzaro N, et al. PBC screen: an IgG/IgA dual i sotype ELISA detecting multiple mitochondrial and nuclear autoantibodies specific for primary biliary cirrhosis. J Autoimmun. (2010) 35:436-42. doi: 10.1016/j.jaut.2010.09.005

15. Norman GL, Yang CY, Ostendorff HP, Shums Z, Lim MJ, Wang J, et al. Antikelch-like 12 and anti-hexokinase 1: novel autoantibodies in primary biliary cirrhosis. Liver Int. (2015) 35:642-51. doi: 10.1111/liv.12690

16. Hu CJ, Song G, Huang W, Liu GZ, Deng CW, Zeng HP, et al. Identification of new autoantigens for primary biliary cirrhosis using human proteome microarrays. Mol Cell Proteomics. (2012) 11:669-80. doi: 10.1074/mcp.M111.015529

17. Gupta VA, Beggs AH. Kelch proteins: emerging roles in skeletal muscle development and diseases. Skeletal Muscle. (2014) 4:11-2. doi: 10.1186/2044-5040-4-11

18. Dhanoa BS, Cogliati T, Satish AG, Bruford EA, Friedman JS. Update on the Kelch-like (KLHL) gene family. Hum Genomics. (2013) 7:13. doi: $10.1186 / 1479-7364-7-13$
19. Robey RB, Hay N. Mitochondrial hexokinases: guardians of the mitochondria. Cell Cycle. (2005) 4:654-8. doi: 10.4161/cc.4.5.1678

20. Cheung K-S, Seto W-K, Fung J, Lai C-L, Yuen M-F. Epidemiology and natural history of primary biliary cholangitis in the Chinese: a territory-based study in Hong Kong between 2000 and 2015. Clin Translat Gastro. (2017) 8:e116. doi: $10.1038 / \operatorname{ctg} .2017 .43$

21. Boonstra K, Beurers U, Ponsioen CY. Epidemiology of primary sclerosing cholangitis and primary biliary cirrhosis: a systematic review. J Hepatol. (2012):1181-8. doi: 10.1016/j.jhep.2011.10.025

22. Lu M, Zhou Y, Haller IV, Romanelli RJ, VanWormer JJ, Rodriguez $\mathrm{CV}$ et al., Increasing Prevalence of primary biliary cholangitis and reduced mortality with treatment. Clin Gast Hepatol. (2018) 16:1342-50. doi: 10.1016/j.cgh.2017.12.033

23. Dahlquist G, Gaouar F, Carrat F, Meurisse S, Chazouilleres O, Poupon R, et al. Large- scale characterization study of patients with antimitochondrial antibodies but nonestablished primary biliary cholangitis. Hepatology. (2017) 65:152-63. doi: 10.1002/hep.28859

24. Prince M, Chetwynd A, Newman W, Metcalf JV, James OF. Survival and symptom progression in a geographically based cohort of patients with primary biliary cirrhosis: follow-up for up to 28 years. Gastroenterology. (2002) 123:1044-51. doi: 10.1053/gast.2002.36027

25. Reig A, Garcia M, Shums Z, Milo J, Encabo S, Bentow C, et al. The Novel Hexokinase (HK-1) antibodies are useful for diagnosis and associated with bad prognosis in primary biliary cholangitis. J Hepatol. (2017) 66(Suppl. 1):S355-6. doi: 10.1016/S0168-8278(17)31049-8

26. Norman GL, Shums Z, Ruiz-Gaspà $S$, Reig $A$, Romera MA, Vi-as $\mathrm{O}$, et al. Similar geographic prevalence of anti-hexokinase and anti-kelch like peptide antibodies in patients with primary biliary cholangitis-A large international multi-center study. In: Conrad K, Andrade LEC, Chan EKL, Fritzler MJ, Pruijn JM, Shoenfeld $\mathrm{Y}$, et al editors. Immunodeficiency, Infection and Autoimmune Diseases. Autoantigens, Autoantibodies, Autoimmunity, Vol. 11. Lengerich: Pabst Science Publishers (2017). p. 459-60.

Conflict of Interest Statement: GN, MM are employees of Inova Diagnostics, Inc.

MF and AP have received speaking and consulting honoraria from Inova Diagnostics, Inc. and Werfen International.

The remaining authors declare that the research was conducted in the absence of any commercial or financial relationships that could be construed as a potential conflict of interest.

Copyright ( 2019 Norman, Reig, Viñas, Mahler, Wunsch, Milkiewicz, Swain, Mason, Stinton, Aparicio, Aldegunde, Fritzler and Parés. This is an open-access article distributed under the terms of the Creative Commons Attribution License (CC BY). The use, distribution or reproduction in other forums is permitted, provided the original author(s) and the copyright owner(s) are credited and that the original publication in this journal is cited, in accordance with accepted academic practice. No use, distribution or reproduction is permitted which does not comply with these terms. 